\title{
TRPV4-Activation Induced Nerve Fibers Injury Mediates the Memory Impairment After Noise Exposure
}

\section{Yang Yang}

Third Military Medical University (Army Medical University)

Ju Wang

Third Military Medical University (Army Medical University)

Chuanyan Yang

Third Military Medical University (Army Medical University)

\section{Yulian Quan}

Third Military Medical University (Army Medical University)

\section{Xuezhu Chen}

Third Military Medical University (Army Medical University)

\section{Xuejiao Lei}

Third Military Medical University (Army Medical University)

\section{Liang Tan}

Third Military Medical University (Army Medical University)

\section{Hua Feng}

Third Military Medical University (Army Medical University)

Fei Li

Third Military Medical University (Army Medical University)

Tunan Chen ( $\boldsymbol{Q}$ ctn@tmmu.edu.cn )

Third Military Medical University (Army Medical University)

\section{Research}

Keywords: Low-frequency noise, Memory impairment, TRPV4, Nerve fibers, Hippocampus

Posted Date: November 22nd, 2021

DOI: https://doi.org/10.21203/rs.3.rs-1061516/v1

License: (1) (1) This work is licensed under a Creative Commons Attribution 4.0 International License. Read Full License 


\section{Abstract}

Background: Excessive exposure to high-intensity, low-frequency noise (HI-LFN) causes vibroacoustic disease (VAD). Memory deficits is one non-auditory symptomatic effect found in humans and rodents with VAD, but the mechanism is largely unknown. This study was designed to explore the nerve fibers impairment in hippocampus and potential mechanism after HI-LFN exposure.

Methods: HI-LFN injury model was established by exposing to noises with a frequency of 100,150 or 200 $\mathrm{Hz}$ and a pressure level of $140 \mathrm{~dB}$. Adult male WT and TRPV4/- mice were employed in present study. After HI-LFN exposure, the new object recognition task and the morris water maze were used for examining the memory impairment, HE staining was used to examine the holistic morphological changes hippocampus, immunofluorescence and western blot were used to the detect the change of nerve fibers in dentate gyrus (DG) and CA1 of the hippocampus.

Results: The expression of TRPV4 was significantly upregulated in hippocampus after HI-LFN exposure. What's more, prominent learning and memory deficits and lower neural cell density with injured nerve fibers in CA1 and DG areas of hippocampus were found in WT mice after HI-LFN exposure. However, $T R P V 4^{-/}$mice showed better performance in learning and memory tests and more integrated nerve fibers in CA1 and DG areas after HI-LFN exposure compared with that in WT mice.

Conclusions: Our data indicated a novel mechanism that the injury of neurons and nerve fibers in hippocampus might be the primary trigger of memory deficits after HI-LFN exposure, and TRPV4 activation plays crucial role in the injury of neurons and nerve fibers in hippocampus, those findings provide a promising therapeutic target for treating cognitive dysfunction after low frequency noise exposure.

\section{Background}

Vibroacoustic disease (VAD) is a cumulative and systemic pathology, which has been identified in aircraft technicians, pilots, cabin crewmembers and disk-jockeys, caused by excessive exposure to high-intensity (over 110 decibels (dB)), low-frequency (blow 200 Hertz (Hz)) noise (HI-LFN)[1]. What's more, VAD has also been observed in several populations exposed to environmental LFN and evidence of the nonauditory symptomatic effects of environmental LFN exposure on public health is increasing. Studies have shown that LFN exposure leads to annoyance, sleep disturbances and impairs cognitive performance in humans[2-4]. Furthermore, long-term exposure to HI-LFN could impair learning and memory abilities in rats[5], and characterized with the activation of glial cells, impaired neurogenesis and neural apoptosis in hippocampus[6, 7]. However, the mechanisms of how the HI-LFN exposure affects the cognitive function are still largely unknown.

Memory formation and forgetting are believed to involve the modifications in neural transmission caused by physiochemical and/or structural modifications of synaptic communication within neuronal network[8], and those processes are strongly mediated by cytoskeleton dynamics, such as actin, 
neurofilament and microtubule[8-10]. Cytoskeletons are important for neuron to maintain the soma and fiber morphology, to support axonal transport and to accommodate shape changes of neural circuits that related to cognitive plasticity[11]. Abnormal cytoskeletons contribute to memory deficit and are found in hippocampus in rodent models of Alzheimer's disease (AD) $[12,13]$. Interestingly, some studies revealed that tissue which are abundant in cytoskeleton are particular targets for HI-LFN, such as the cochlear stereocilia and respiratory tract brush cell microvilli[14, 15], indicating that cytoskeletons in hippocampus are potential target for HI-LFN and nerve fibers injury based on cytoskeletons injury is responsible for learning and memory impairment after HI-LFN exposure.

Transient receptor potential vanilloid sub type 4 (TRPV4) is a member of TRP super family of $\mathrm{Ca}^{2+}$ permeable non-selective cation channels[16]. Functionally, TRPV4 is involved in cellular processes such as mechanosensation, osmosensation and thermosensation[17]. Interestingly, one study demonstrated that TRPV4 physically interacts with tubulin, actin and neurofilament proteins to influence cellular cytoskeletal dynamics and mechanosensitive processes[17]. Thereby it can integrate signaling of various intracellular second messengers and signaling cascades in response to external mechanical stimuli. What's more, previous studies have suggested that TRPV4 channel might be involved in translating intracellular biological signaling of acoustic stimuli[7, 18, 19]. Therefore, we speculated that as a type of acoustic stimulus, HI-LFN exposure impairs cellular cytoskeleton and nerve fibers via TRPV4 channel.

In the present study, we explored the TRPV4-activation induced neurons and nerve fibers injury in hippocampus mediates the memory impairment after HI-LFN exposure in mice. Our data indicated that the expression of TRPV4 in hippocampus was upregulated after HI-LFN exposure. What's more, the wild type (WT) mice after HI-LFN exposure developed significant learning and memory deficit with lower pyramidal and granular cell density and injured nerve fibers in hippocampal CA1 and dentate gyrus (DG) area. However, $\mathrm{TRPV4}^{-{ }^{--}}$mice showed better performance in learning and memory tests and more integrated nerve fibers in hippocampus after HI-LFN exposure compared with that in WT mice. In summary, these findings suggested the crucial role of TRPV4 activation in nerve fibers injury and memory impairment after HI-LFN exposure, which could provide novel insight for the therapeutic development.

\section{Methods}

\section{Animals}

The TRPV4 ${ }^{-/}$mice were established from Cyagen Biosciences Inc. Adult male wild type (WT) and TRPV4 ${ }^{-/}$mice (8-10 weeks, $20-25 \mathrm{~g}$ ) in same litter were used in this study. The mice were housed in a specific temperature-controlled room under a standard 12-h light/12-h dark cycle and provided free access to food and water. All experiments are reported in compliance with the Animal Research: Reporting in Vivo Experiments (ARRIVE) guidelines. Our experimental protocols were approved by the Laboratory Animal Welfare and Ethics Committee of the Third Military Medical University (AMUWEC20210680) and performed according to the Guide for the Care and Use of Laboratory Animals. Randomization was carried out using odd/even numbers. 


\section{The noise device and exposure condition}

The noise device used in this paper as previous paper described[20]. Noise with a frequency of 100, 150 or $200 \mathrm{~Hz}$ and a pressure level of $140 \mathrm{~dB}$ was used in this study. The animals were exposed with noises with different frequency and same pressure, those processes lasted for 2 hours for 3 consecutive days. Then, the animals were used for behavioral tests, or the brains were collected for morphological and biochemical experiments after deeply anesthetized.

\section{Western blot analysis}

Western blot (WB) was performed as described by Yang et al[21]. The brains were obtained after noise exposure, then the protein of hippocampus tissues was sampled. The primary antibodies included rabbit anti-transient receptor potential vanilloid 4 (TRPV4) polyclonal antibody (1:1000; Abcam), rabbit antineurofilament 200 (NF200) polyclonal antibody (1:1000; Sigma), goat anti-myelin basic protein (MBP) polyclonal antibody (1:1000; Santa Cruz Biotechnology), mouse anti- $\beta$-actin (1:1000; Boster, Wuhan, China) and mouse anti-glyceraldehyde3-phosphate dehydrogenase (GAPDH, 1:2000, Boster) for $12 \mathrm{~h}$ at 4 ${ }^{\circ} \mathrm{C}$. The secondary antibody was anti mouse, goat or rabbit IgG HRP conjugated (1:10000; Abcam). Finally, an enhanced chemiluminescence reagent kit (Millipore, Temecula, CA, USA) for western blotting was used to visualize the immunoreactive bands, the relative densities of the bands were analyzed using Image Lab software.

\section{Immunofluorescence and HE staining}

Immunofluorescence was performed according to the method described by Yang et al[22]. Thirtymicrometer-thick cryostat sections were incubated with the following primary antibodies in $1 \%$ BSA (overnight, $4{ }^{\circ} \mathrm{C}$ ): rabbit anti- NF200 polyclonal antibody (1:1000; Sigma), goat anti-MBP polyclonal antibody (1:1000; Santa). After the sections were washed with $0.01 \mathrm{M}$ phosphate buffer, they were probed with the appropriate Cy3-, 488-conjugated and Cy3-SABC secondary antibodies (1:500, $4 \mathrm{~h}$, at room temperature (RT); Jackson ImmunoResearch). Finally, the sections were mounted in Vectashield medium (Vector Laboratories). Hematoxylin-eosin (HE) was performed according to our previous study[23]. After deparaffinized in xylene and rehydrated through graded alcohol, the five-micrometer-thick paraffin sections were incubated by HE reagents. The stained cells were viewed and photographed under a Zeiss (Oberkochen, Germany) Axivert microscope equipped with a Zeiss AxioCam digital color camera connected to the Zeiss AxioVision 3.0 system.

\section{Behavioral tests}

The new object recognition (NOR) task: Mice were acclimatized to the experimental room for one night prior to each test. The NOR task was performed as previously described with minor modification[24]. Mice were conducted in an open field box located in a soundattenuated room to assess short-term memory. Briefly, after a 5-min habituation period, the mice were tested in the first trial: two identical cylinders were employed in diagonal corners, and each mouse was placed in the box and allowed to explore freely the 
objects for $10 \mathrm{~min}$. In the second trail, one of the cylinders was replaced with a new cuboid object, and the time for exploring freely was $10 \mathrm{~min}$. The 2 trials were separated by $10 \mathrm{~min}$. Only the second trial (retention session) was analyzed, and discrimination index defined as the ratio of the time spent exploring the cuboid object to the total time spent exploring both objects. Individual movement tracks were analyzed by Ethovision 10.0 (Noldus).

Morris water maze (MWM): MWM was performed as previous Study[25]. Briefly, the water maze is equipped with a $1.3 \mathrm{~m}$ diameter white pool and a $10 \mathrm{~cm}$ diameter hidden platform which is submerged approximately $1 \mathrm{~cm}$ below the water level. The mice were trained with 4 trails (one trail mean that the mouse is released into the water at water-level from one quadrant for exploring $1 \mathrm{~min}$ ) per day for 5 days to find and remember the hidden platform. On the $7^{\text {th }}$ day, the animals were given a probe trial. The platform was removed, and the mice were allowed to explore the platform location for 60 seconds. During the probe trial, the platform was moved, the number of times the animal crossed the location of the hidden platform and the duration of time in each quadrant was calculated. All the trails were recorded with a ceiling-mounted video camera and analyzed by Ethovision 10.0 (Noldus).

\section{Statistical analysis}

All the data collected in a double-blind manner and statistical analyses were performed using SPSS 18.0 software. Data were presented as mean \pm SEM and analyzed using the paired or unpaired Student's t-test or analysis of variance (ANOVA). A Bonferroni test was used as a post-hoc test following ANOVA. $P<0.05$ was considered to indicate a statistically significant difference.

\section{Results}

\section{HI-LFN exposure increased the expression of TRPV4}

The WT mice were exposed to $140 \mathrm{~dB}$ noise at 100,150 or $200 \mathrm{~Hz}$ for 2 hours for 3 consecutive days. We found that the expression of TRPV4 in hippocampus tissue was significantly increased after HI-LFN exposure (Fig. 1A-B, $P<0.05$ ) compared with that in control group, and TPRV4 expression was highest when exposed to $200 \mathrm{~Hz}$ noise (Fig. $1 \mathrm{~A}-\mathrm{B}, \mathrm{P}<0.001$ ). We next used the mice in $140 \mathrm{~dB}, 200 \mathrm{~Hz}$ group to analyze the morphological changes and functional tests of hippocampus.

\section{TRPV4 knockout rescued the memory impairment induced by HI-LFN exposure}

To investigate the function of TRPV4 overexpression in hippocampus after noise exposure, we developed TRPV4 ${ }^{-/-}$mice. Behaviorally, we used the new object recognition (NOR) task and the morris water maze (MWM) to assess the memory impairment of the WT and TRPV4 ${ }^{-/-}$mice after $140 \mathrm{~dB}, 200 \mathrm{~Hz}$ noise exposure. What's more, the NOR task is used to evaluate recognition memory and short-term memory, the MWM is designed to test spatial memory and long-term memory, both the NOR task and the MWM were usually used to assess the cognitive function of hippocampus. In the NOR task, there were no difference in total distance moved (Fig. 2B, $\mathrm{P}>0.05$ ) and total exploration time of the two objects (Fig. 2C, $\mathrm{P}>0.05$ ) 
between the WT and TRPV4 ${ }^{-/-}$mice with or without HI-LFN exposure, indicating that the results are not influenced by noise exposure in overall activity or lack of motivation. Furthermore, in contrast to the WT mice without HI-LFN exposure, HI-LFN exposure induced the WT mice a significant deficit in recognition memory with decreased exploring time of novel object (Fig. 2D, P<0.01). However, after HI-LFN exposure, the $T R P V 4^{-/-}$mice exhibited a significant preference for the novel object (Fig. 2D, $\left.\mathrm{P}<0.05\right)$ compared with that in the WT group. Similarly, in the MWM test, in contrast to the WT mice without HI-LFN exposure, HILFN exposure induced the WT mice a significant delay for exploring the platform at the day 5 during training trail (Fig. 2E-F, $P<0.01$ ), less exploring time in target quadrant (Fig. $2 \mathrm{E}$ and $\mathrm{G}, P<0.01$ ) and less number of crossing annulus (Fig. $2 \mathrm{E}$ and $\mathrm{H}, P<0.01$ ) during probe trail. Nevertheless, under HI-LFN exposure condition, the $T R P V 4^{-/-}$mice exhibited a significant short time for exploring the platform at the day 5 during training (Fig. 2E-F, $\mathrm{P}<0.05$ ), more exploring time in target quadrant (Fig. 2E and $\mathrm{G}, P<0.05$ ) and increased number of crossing annulus ( $F i g .2 \mathrm{E}$ and $\mathrm{H}, P<0.05$ ) during probe trail compared with that in the WT group. Above results indicated that TRPV4 overexpression mediated the memory deficit after noise exposure and TRPV4 knockout alleviated the memory impairment.

\section{TRPV4 knockout alleviated the impairment of pyramidal cells and granular cells in hippocampus induced by HI-LFN exposure}

Next, HE staining was used to analyze the morphological changes of pyramidal cell layer (PCL) of CA1 and granular cell layer (GCL) of DG in hippocampus which play important role in learning and memory[26]. Interestingly, in contrast to the WT mice without HI-LFN exposure, HI-LFN exposure induced a significant decrease in the thickness of cellular layers in PCL and GCL (Fig. 3A-B and D, $P<0.01$ ). What's more, the number of pyramidal neurons in PCL and granular neurons in GCL per $100 \mu \mathrm{m}$ in HI-LFN group were also apparently decreased compared with that in the WT group (Fig. 3A, C and E, $P<0.01$ ). However, under HI-LFN exposure condition, TRPV4 knockout significantly alleviated the PCL and GCL injury in thickness and neural density (Fig. $3 A-E, P<0.05)$ compared that in the WT group.

\section{TRPV4 knockout alleviated the nerve fibers injury in hippocampus induced by HI-LFN exposure}

The memory function of hippocampus mediated by neural circuits which are composed by massive nerve fibers. NF200 is a marker for neurofilaments, the neurofilaments are believed to function primarily to provide structural support for axons. MBP is marker for myelin membrane and believed to be important in the process of myelination of nerves. We labelled the nerve fibers with NF200 and MBP antibodies to observe the morphological change of neural circuits in CA1 and DG after HI-LFN exposure. We found that HI-LFN exposure induced disordered arranged nerve fibers (Fig. 4A) of pyramidal cells in CA1 and granular cells in DG, and a significant decreased protein expression level of NF200 and MBP (Fig. 4B, $P<0.01)$ in hippocampus in the WT mice. Interestingly, TRPV4 knockout rescued the nerve fibers injury and increased the expression level of NF200 and MBP (Fig. 4A-B, P<0.05) in CA1 and DG areas compared that in the WT group under HI-LFN exposure condition, indicating that TRPV4 overexpression induced nerve fibers injury in hippocampus might mediated memory impairment after HI-LFN exposure. 


\section{Discussion}

In the present study, we investigated the TRPV4-activation induced neurons and nerve fibers injury in hippocampus mediates the memory impairment after HI-LFN exposure in mice. The results indicated that the expression of TRPV4 was upregulated in hippocampus after HI-LFN exposure. What's more, significant learning and memory deficits with lower neuron density and injured nerve fibers in hippocampus were found in WT mice after HI-LFN exposure. However, TRPV4 ${ }^{-/-}$mice showed better performance in learning and memory tests and more integrated nerve fibers in hippocampus after HI-LFN exposure compared with that in WT mice.

It was well documented that long-term exposure to HI-LFN could impair learning and memory abilities[2, 5], but the mechanisms of how the HI-LFN exposure affects the cognitive function are still largely unknown. Studies showed that activation of glial cells, injured brain blood barrier, and impaired neurogenesis in hippocampus might contribute to the cognitive deficits after HI-LFN exposure[5, 6, 20, 27]. Anatomically, the hippocampus is composed of several regions and connected with a lot of brain regions, thousands of nerve fibers and synaptic connections are assembled with these complex circuits for memory formation and storage[28, 29]. Interestingly, the decreased pyramidal neurons and granular neurons density with less the nerve fibers were detected in the CA1 and DG of hippocampus after HI-LFN exposure in present work, indicating the neural circuits were damaged. What's more, several mnemonic functions have been identified to be closely related with the CA1 in hippocampus, such as novelty detection[30], binding of information to spatial contexts[31] and working memory[32]. Behaviorally, we found the mice developed significant mnemonic functions impairment in the new object recognition task and the morris water maze after HI-LFN exposure. Thus, we think that the injury of neural circuits and neurons in hippocampus might be the primary triggers of memory deficits after noise exposure.

Mechanotransduction is a fundamental biological process in sensing and responding to the physical environment for cells[33]. Cells express a lot of channels in membrane for sensing mechanical inputs, the extensively documented mechanically activated ion channels are PIEZOs and TRPV4[34]. These channels could be activated by a diverse range of physical forces such as vibration, stretch, or sound waves, and transduce the mechanical inputs into biochemical signals. Previous study revealed that TRPV4 was activated in glial cells in hippocampus, then induced learning and memory deficits after noise exposure. Similarly, our results showed that the expression of TRPV4 was significantly increased after HI-LFN exposure. However, TRPV4 is also expressed in hippocampal neurons which are abundant of massive cytoskeletons and formed complex neural circuits, and the neural cytoskeletons work as major players in molecular-level processing of information required for learning and memory storage[11, 26, 35, 36]. Additionally, some suggestive evidences demonstrated that TRPV4 physically interacts with cytoskeleton proteins to influence mechanosensitive processes[17] and tissues which are abundant in cytoskeleton are special targets for HI-LFN $[14,15]$. Therefore, we think that TRPV4 activation in pyramidal and granular neurons directly induces cytoskeletons injury, and then causes nerve fibers impairment and memory deficits after HI-LFN exposure. Fortunately, we really found more integrated structure of nerve fibers in

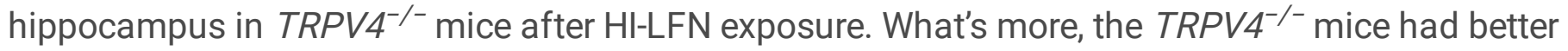


performance in memory tests than WT mice after noise exposure. Those results suggested a crucial role of TRPV4 activation in nerve fibers injury and memory impairment after HI-LFN exposure. Additionally, and the results should be verified in conditional knockout mice in the future.

\section{Conclusion}

In summary, our data indicated a novel mechanism that the injury of neural circuits in hippocampus might be the primary trigger of memory deficits after HI-LFN exposure, and TRPV4 activation plays crucial role in the injury of neurons and nerve fibers in hippocampus. Our findings provide a promising therapeutic candidate for treating cognitive dysfunction of vibroacoustic disease after low frequency noise exposure.

\section{Abbreviations}

AD: Alzheimer's disease; dB: decibels; CA1: field CA1 of hippocampus; PCL: pyramidal cell layer; Hz: Hertz; HI-LFN: high-intensity, low-frequency noise; MBP: myelin basic protein; MWM: morris water maze; NF200: neurofilament 200; NOR: new object recognition; TRPV4: transient receptor potential vanilloid 4; VAD: Vibroacoustic disease; WT: wild type.

\section{Declarations}

\section{Acknowledgements}

Not applicable.

\section{Authors' contributions}

H.F., T.C., and F.L. designed the experiments. J.W., X.L., L.T., Y.Q., C.Y. and X.C. preformed the experiments and discussed the results. Y.Y., J.W. and C.Y. collected and analyzed all the present data. F.L. and T.C. wrote the draft and worked on the manuscript revision.

\section{Funding}

The present study was funded by the National Natural Science Foundation of China (Grant No. 81901267) and the Southwest Hospital (Grant no. SWH2017JSZD-10).

\section{Availability of data and materials}

The datasets used and/or analysed during the current study are available from the corresponding author on reasonable request.

\section{Ethics approval and consent to participate}


The experimental protocols were approved by the Ethics Committee of the Third Military Medical University.

\section{Consent for publication}

All authors read and approved the final manuscript.

\section{Competing interests}

The authors declare that they have no competing interests.

\section{Author details}

1 Department of Neurosurgery and Key Laboratory of Neurotrauma, Southwest Hospital, Third Military Medical University (Army Medical University), Chongqing 400038, China.

2 F.M. Kirby Neurobiology Center, Boston Children's Hospital, Harvard Medical School, Boston, MA, USA.

\section{References}

1. Branco, N.A. and M. Alves-Pereira, Vibroacoustic disease. Noise Health, 2004. 6(23): p. 3-20.

2. Gomes, L.M., A.J. Martinho Pimenta, and N.A. Castelo Branco, Effects of occupational exposure to low frequency noise on cognition. Aviat Space Environ Med, 1999. 70(3 Pt 2): p. A115-8.

3. Tzivian, L., et al., Long-Term Air Pollution and Traffic Noise Exposures and Mild Cognitive Impairment in Older Adults: A Cross-Sectional Analysis of the Heinz Nixdorf Recall Study. Environ Health Perspect, 2016. 124(9): p. 1361-8.

4. Tzivian, L., et al., Associations of long-term exposure to air pollution and road traffic noise with cognitive function-An analysis of effect measure modification. Environ Int, 2017. 103: p. 30-38.

5. Shi, M., et al., Glial cell-expressed mechanosensitive channel TRPV4 mediates infrasound-induced neuronal impairment. Acta Neuropathol, 2013. 126(5): p. 725-39.

6. Cai, J., et al., Epigallocatechin gallate (EGCG) attenuates infrasound-induced neuronal impairment by inhibiting microglia-mediated inflammation. J Nutr Biochem, 2014. 25(7): p. 716-25.

7. Lehnert, B.P., et al., Distinct roles of TRP channels in auditory transduction and amplification in Drosophila. Neuron, 2013. 77(1): p. 115-28.

8. Lamprecht, R., The roles of the actin cytoskeleton in fear memory formation. Front Behav Neurosci, 2011. 5: p. 39.

9. Craddock, T.J., J.A. Tuszynski, and S. Hameroff, Cytoskeletal signaling: is memory encoded in microtubule lattices by CaMKII phosphorylation? PLoS Comput Biol, 2012. 8(3): p. e1002421.

10. Davis, R.L. and Y. Zhong, The Biology of Forgetting-A Perspective. Neuron, 2017. 95(3): p. 490-503.

11. Baas, P.W., et al., Stability properties of neuronal microtubules. Cytoskeleton (Hoboken), 2016. 73(9): p. 442-60. 
12. Ballatore, C., et al., Microtubule stabilizing agents as potential treatment for Alzheimer's disease and related neurodegenerative tauopathies. J Med Chem, 2012. 55(21): p. 8979-96.

13. Brunden, K.R., et al., The characterization of microtubule-stabilizing drugs as possible therapeutic agents for Alzheimer's disease and related tauopathies. Pharmacol Res, 2011. 63(4): p. 341-51.

14. Alves-Pereira, M., Noise-induced extra-aural pathology: a review and commentary. Aviat Space Environ Med, 1999. 70(3 Pt 2): p. A7-21.

15. Castelo Branco, N.A., et al., [Respiratory epithelia in Wistar rats born in low frequency noise plus varying amounts of additional exposure]. Rev Port Pneumol, 2003. 9(6): p. 481-92.

16. Everaerts, W., B. Nilius, and G. Owsianik, The vanilloid transient receptor potential channel TRPV4: from structure to disease. Prog Biophys Mol Biol, 2010. 103(1): p. 2-17.

17. Goswami, C., et al., Importance of non-selective cation channel TRPV4 interaction with cytoskeleton and their reciprocal regulations in cultured cells. PLoS One, 2010. 5(7): p. e11654.

18. Kim, C., TRPV Family Ion Channels and Other Molecular Components Required for Hearing and Proprioception in Drosophila, in TRP Ion Channel Function in Sensory Transduction and Cellular Signaling Cascades, W.B. Liedtke and S. Heller, Editors. 2007: Boca Raton (FL).

19. Kim, J., et al., A TRPV family ion channel required for hearing in Drosophila. Nature, 2003. 424(6944): p. $81-4$.

20. Wang, X., et al., Effect of low-frequency but high-intensity noise exposure on swine brain blood barrier permeability and its mechanism of injury. Neurosci Lett, 2018. 662: p. 122-128.

21. Yang, Y., et al., Epothilone B Benefits Nigrostriatal Pathway Recovery by Promoting Microtubule Stabilization After Intracerebral Hemorrhage. J Am Heart Assoc, 2018. 7(2).

22. Yang, Y., et al., SVCT2 Promotes Neural Stem/Progenitor Cells Migration Through Activating CDC42 After Ischemic Stroke. Front Cell Neurosci, 2019. 13: p. 429.

23. Yang, Y., et al., Quantitative Iron Neuroimaging Can Be Used to Assess the Effects of Minocycline in an Intracerebral Hemorrhage Minipig Model. Transl Stroke Res, 2020. 11(3): p. 503-516.

24. Zhang, Q., et al., Citalopram restores short-term memory deficit and non-cognitive behaviors in APP/PS1 mice while halting the advance of Alzheimer's disease-like pathology. Neuropharmacology, 2018. 131: p. 475-486.

25. Vorhees, C.V. and M.T. Williams, Morris water maze: procedures for assessing spatial and related forms of learning and memory. Nat Protoc, 2006. 1(2): p. 848-58.

26. Hainmueller, T. and M. Bartos, Dentate gyrus circuits for encoding, retrieval and discrimination of episodic memories. Nat Rev Neurosci, 2020. 21(3): p. 153-168.

27. Shi, Y.J., et al., Inhibitive Effects of FGF2/FGFR1 Pathway on Astrocyte-Mediated Inflammation in vivo and in vitro After Infrasound Exposure. Front Neurosci, 2018. 12: p. 582.

28. Cacucci, F., P. Salinas, and T.J. Wills, Hippocampus: Activity-Driven Maturation of Neural Circuits for Navigation. Curr Biol, 2017. 27(11): p. R428-R430. 
29. Lisman, J.E., Relating hippocampal circuitry to function: recall of memory sequences by reciprocal dentate-CA3 interactions. Neuron, 1999. 22(2): p. 233-42.

30. Hunsaker, M.R., J.S. Rosenberg, and R.P. Kesner, The role of the dentate gyrus, CA3a,b, and CA3c for detecting spatial and environmental novelty. Hippocampus, 2008. 18(10): p. 1064-73.

31. Lee, J.W. and M.W. Jung, Separation or binding? Role of the dentate gyrus in hippocampal mnemonic processing. Neurosci Biobehav Rev, 2017. 75: p. 183-194.

32. Sasaki, T., et al., Dentate network activity is necessary for spatial working memory by supporting CA3 sharp-wave ripple generation and prospective firing of CA3 neurons. Nature Neuroscience, 2018. 21(2): p. 258-269.

33. Ranade, S.S., R. Syeda, and A. Patapoutian, Mechanically Activated lon Channels. Neuron, 2015. 87(6): p. 1162-1179.

34. Martinac, B. and K. Poole, Mechanically activated ion channels. Int J Biochem Cell Biol, 2018. 97: p. 104-107.

35. Lipski, J., et al., Involvement of TRP-like channels in the acute ischemic response of hippocampal CA1 neurons in brain slices. Brain Res, 2006. 1077(1): p. 187-99.

36. Priel, A., J.A. Tuszynski, and N.J. Woolf, Neural cytoskeleton capabilities for learning and memory. J Biol Phys, 2010. 36(1): p. 3-21.

\section{Figures}

A
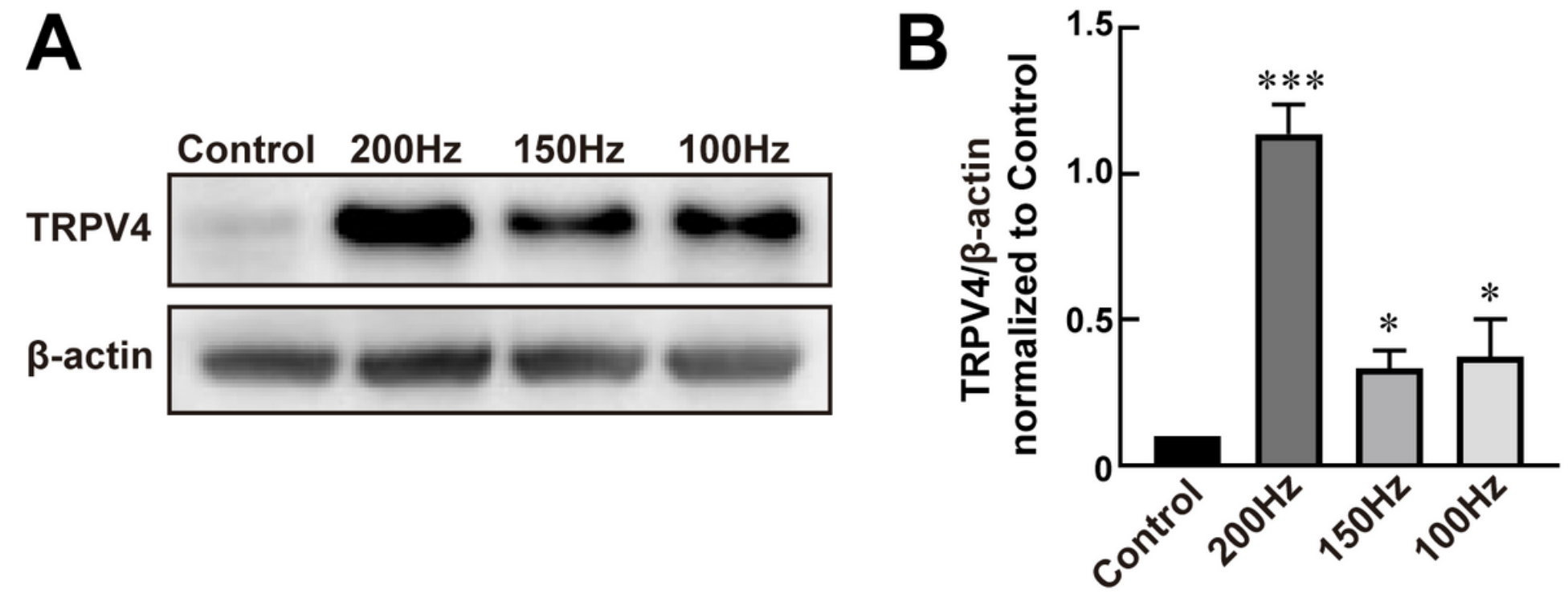

\section{Figure 1}

The expression of TRPV4 after HI-LFN exposure. A-B, Western blot photographs (A) and quantitative analysis (B) of TRPV4 and $\beta$-actin in Control, $200 \mathrm{~Hz}, 150 \mathrm{~Hz}$ and $100 \mathrm{~Hz}$ groups. Data are shown as mean 
\pm SEM, one-way ANOVA, followed by Bonferroni post hoc test $(n=5$ for each group; $* \star \star P<0.001, * P<0.05$ vs Control group).
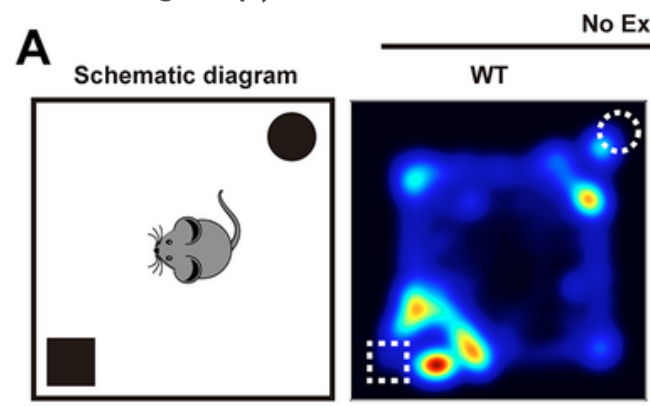

No Exposure
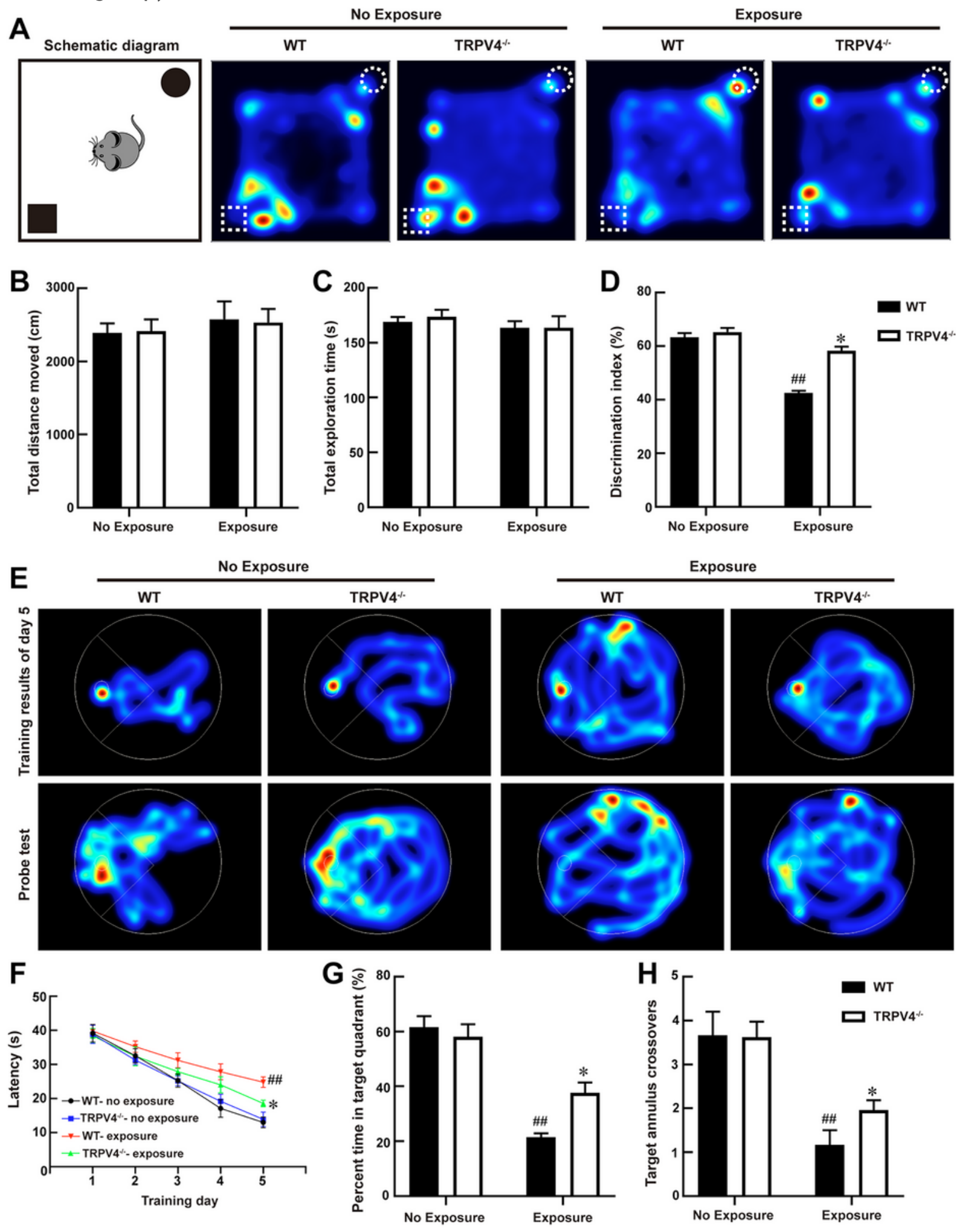

Figure 2

Learning and memory tests of WT and TRPV4-/- mice after HI-LFN exposure. A, The schematic diagram and representative hot maps of the novel object recognition (NOR) task in WT and TRPV4-/- groups with or without HI-LFN exposure. B-D, Quantitative data of total distance moved (B), total exploration time of 
the two objects(C) and discrimination index of the two objects (D) during the NOR task in WT and TRPV4-/- groups with or without HI-LFN exposure. E, Representative morris water maze (MWM) spatial learning at day 5 (up) and memory (down) images. F-H, Quantitative date of latency for 5 days training $(F)$, the percent time in target quadrant $(G)$ and number of target annulus crossovers $(H)$ during MWM test in WT and TRPV4-/- groups with or without HI-LFN exposure. Data are shown as mean \pm SEM, paired or unpaired Student's t-test (two-tailed; B-D, G-H) and two-way ANOVA (F) followed by Bonferroni post hoc test for multiple comparisons ( $n=8$ for each group; \#\#P<0.01 vs WT-no exposure group, ${ }^{\star} \mathrm{P}<0.05$ vs WTexposure group).

A

CA1

Pyramidal cell layer(PCL)

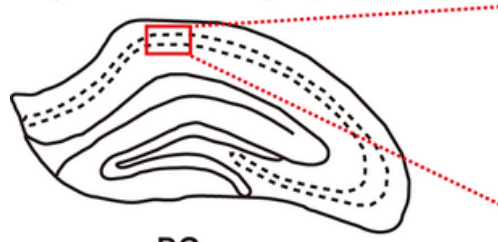

DG

Granular cell layer(GCL)
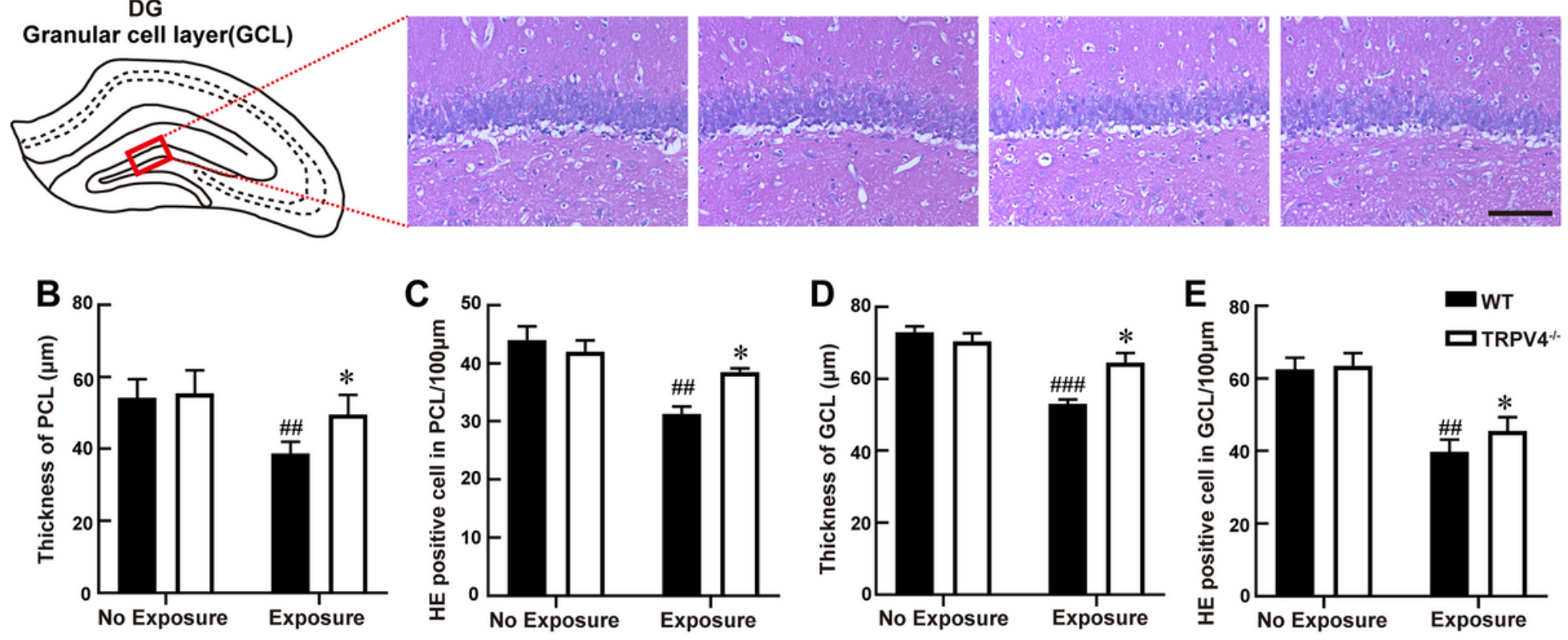

Figure 3

TRPV4 knockout alleviated the impairment of pyramidal cell layer (PCL) and granular cell layer (GCL) induced by HI-LFN exposure. A, Representative HE staining images of PCL of CA1 and GCL of the dentate gyrus (DG) in WT and TRPV4-/- groups with or without HI-LFN exposure (Scale bar: $100 \mu \mathrm{m}$ ). B,

Quantitative data of the thickness of PCL for the four groups. C, Quantitative data of the HE positive neurons in PCL per $100 \mu \mathrm{m}$ for the four groups. D, Quantitative data of the thickness of GCL for the four groups. E, Quantitative data of the HE positive neurons in GCL per $100 \mu \mathrm{m}$ for the four groups. Data are shown as mean \pm SEM, paired or unpaired Student's t-test (two-tailed) $(n=5$ for each group; \#\#P<0.01 and $\# \# \# \mathrm{P}<0.001$ vs $\mathrm{WT}$-no exposure group, ${ }^{*} \mathrm{P}<0.05$ vs WT-exposure group). 


\section{A}
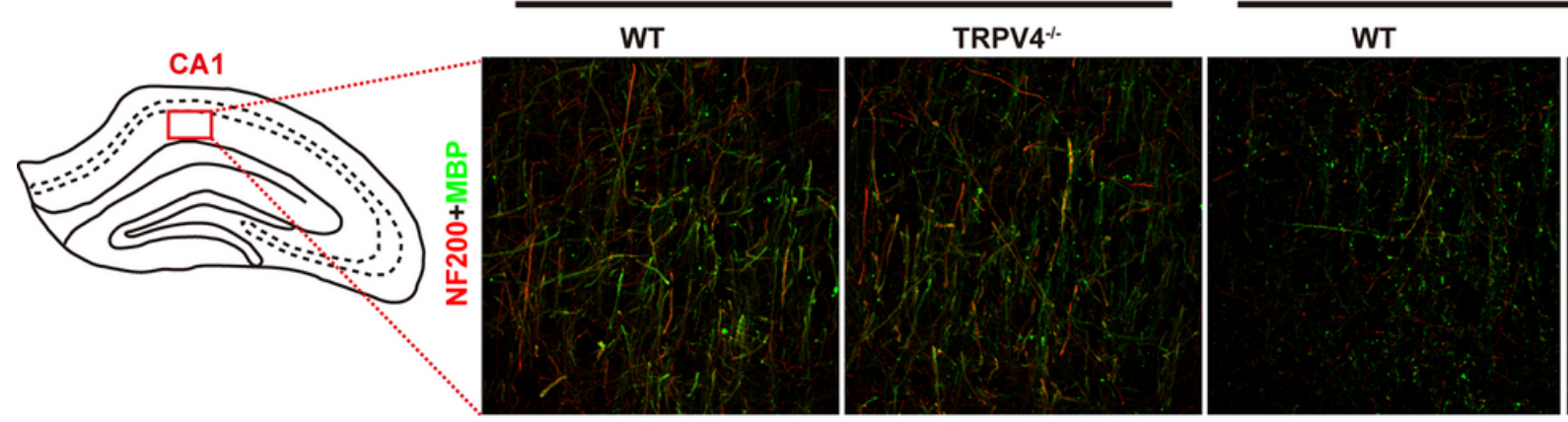

Exposure
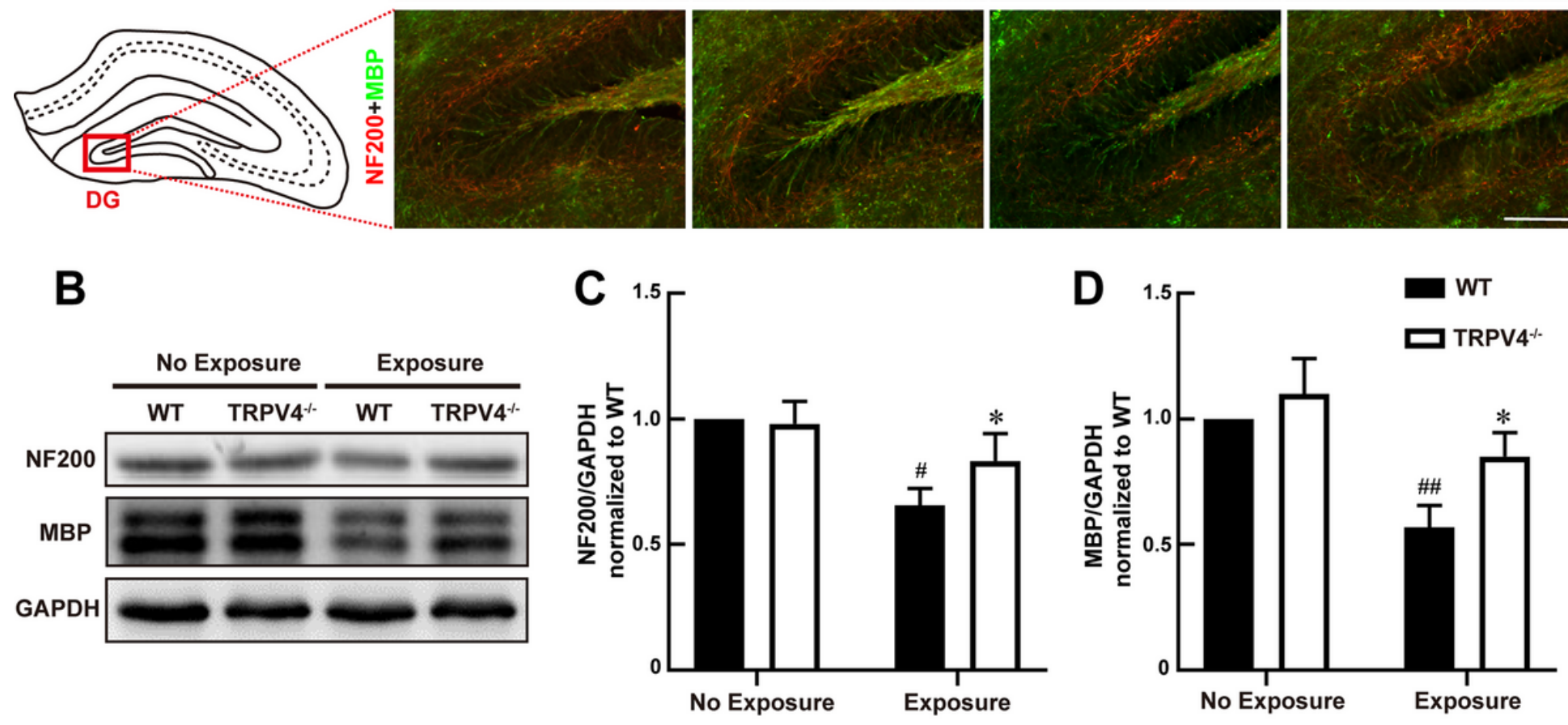

Figure 4

TRPV4 knockout alleviated the nerve fibers injury in hippocampus induced by HI-LFN exposure. A, Representative double immunofluorescence staining of NF200 and MBP of the CA1 and DG area in WT and TRPV4-/- groups with or without HI-LFN exposure (Scale bar: $20 \mu \mathrm{m}$ (up) and $100 \mu \mathrm{m}$ (down)). B, Western blot photographs and quantitative analysis NF200, MBP and GAPDH of the DG area in WT and TRPV4-/- groups with or without HI-LFN exposure. Data are shown as mean \pm SEM, paired or unpaired Student's t-test (two-tailed) ( $\mathrm{n}=5$ for each group; \#\#P<0.05 and \#\#P<0.01 vs WT-no exposure group, *P<0.05 vs WT-exposure group). 